\title{
Adaptive Surface Ship-Wake Detection Based on Improved One-Class Support Vector Machine
}

\author{
Cheng Wang ${ }^{1, a}$, Tingfei Yang ${ }^{1, b}$, Qiang Meng ${ }^{1, c}$ \\ ${ }^{1}$ Navigation Institute. Northwestern Polytechnical University, China \\ achwang@nwpu.edu.cn, byangtff@163.com, mq0539@mail.nwpu.edu.cn
}

Keywords: mathematic wake echo signal model; One-class support vector machine; Sequential minimal optimization; ship-wake detector.

Abstract. It is difficult to collect bubble-wake signals from different ocean environments caused by various types of ship. One-class support vector machine (OCSVM) can make decision based on incomplete information. This paper found an OCSVM detection model which use only reflected signals without a bubble wake to detect the surface ship-wake. In order to improve the training efficiency, a training algorithm based on Sequential Minimal Optimization (SMO) was introduced for OCSVM. Grid search method and Particle Swarm Optimization (PSO) algorithm are used to search optimal parameter. The simulation shows that the proposed detector can detect the ship weak well and it was robust with respect to noisy signals.

\section{Introduction}

Ship's wake can be detected and tracked effectively by active sonar systems with high-frequency signals based on the existence of ship wake bubbles, which can remain for a long time after a ship has left the area. The accuracy of the wake echo signal model has a great impact on the study of processing and detecting method of the ship wake echo signal. A modeling and simulating methods of the mathematic wake echo signal designed based on actual physical model was building in reference [1]. An one-class support vector machine has property to plausibly design an optimal detector for discriminating between two different reflected signals ${ }^{[2]}$. Based on properly describing one-class data distribution in the feature space, Sungmoon Jeong et al proposed a surface ship-wake detector using active sonar and one-class support vector machine ${ }^{[3]}$.

In this paper, we generate a wake echo signal model and use an adaptive method to extract features of the signal. An OCSVM model based on PSO was used to design a detector. It is proved that this method has good ability and high robustness.

\section{Wake Generate}

Simulating the ship wake echo signal include two works to complete: simulate narrow-band noise as the carrier wave, and the slowly varying envelope curve of the signal. According to the FOM reverberation statistical model, the instantaneous value of the ship wake echo signal obeys a Gaussian distribution, while probability density function of the signal envelope is logarithmic Rayleigh distribution ${ }^{[1]}$.

Under the assumption that the velocity of the ship is $16 \mathrm{kn}$, the temperature of the sea is $20^{\circ} \mathrm{c}$ and the salinity is $25 \%_{0}$, the sound speed $c$ equals $1511 \mathrm{~m} / \mathrm{s}$ and the attenuation coefficient $\beta$ equals $0.1 \mathrm{~dB} / \mathrm{m}$. And transmission interval $\tau$ is $10 \mathrm{~ms}$. Assume that transmitting and receiving transducer doesn't have any directionality, within the range of primary maximum the value of band b' are 1 , they all equal to 0 beyond the range. Assume the sonar sensor system is installed at $20 \mathrm{~m}$ below the ocean, and the thickness of the wake is $5 \mathrm{~m}$. We get the narrow-band carrier signal by filtering the white gauss noise with a Gaussian band-pass filter with the order of 165, the center frequency of which is $400 \mathrm{kHz}$ and the band width is $20 \mathrm{kHz}$.

When the envelope of the ship wake echo signal and the narrow-band carrier signal pass through the multiplier, we got the echo signal of ship wake in Fig. 1. 


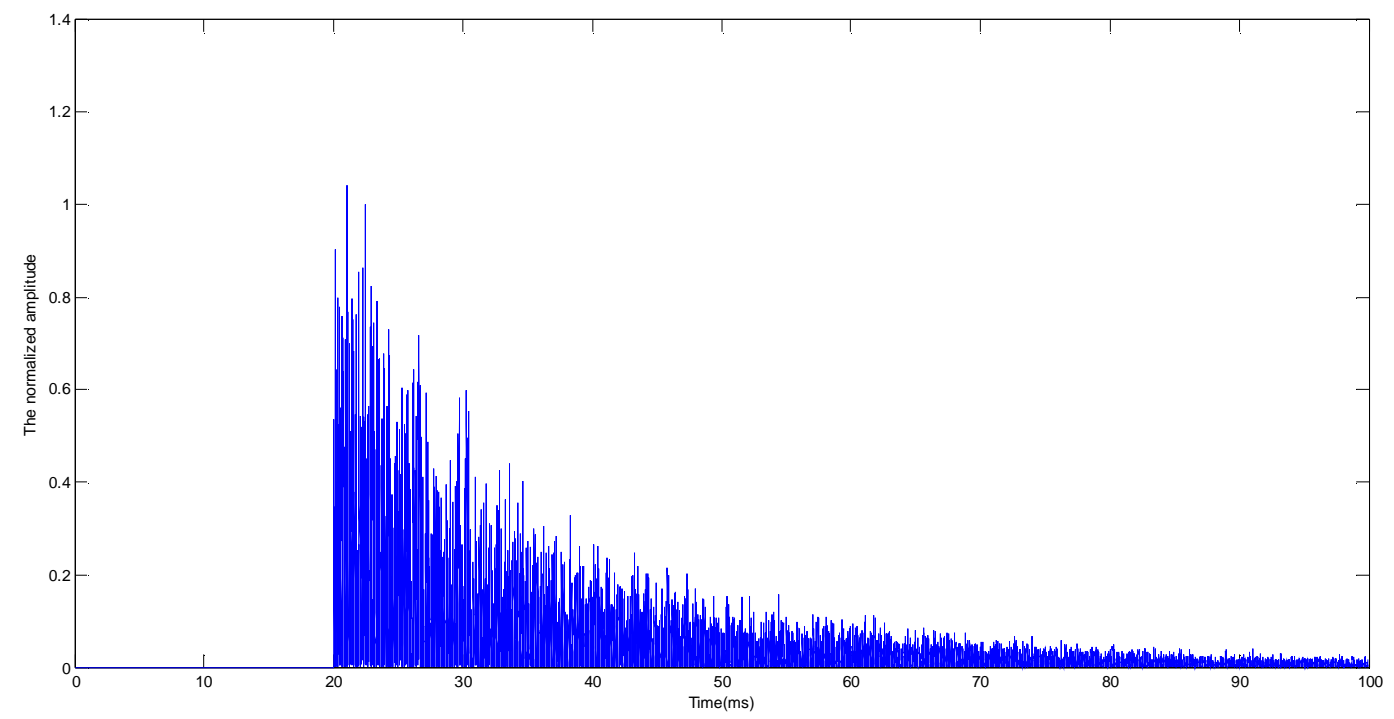

Fig. 1. Echo signal of ship wake

\section{Adaptive Feature Extraction}

A Gaussian finite impulse response (FIR) filter is applied to the received signals to remove the ambient noises. We extracted two features from the received signals, which are then used as the input feature for the detector: a time interval satisfying the bubble-wake effects which is called as the region-of-interest (ROI) and the total average of the amplitude within the ROI time interval. As Fig. 2 shows, the ROI time interval is generated from the initial time point Tmin to the final time point Tmax by counting the number of signal amplitudes without bubble-wake effects. Figs. 3 and 4 show the ROI time interval and the average amplitude of the signals during the ROI time interval when using the three different fixed ROI time intervals 10, 30, and 20ms, and the adaptively obtained ROI time interval.
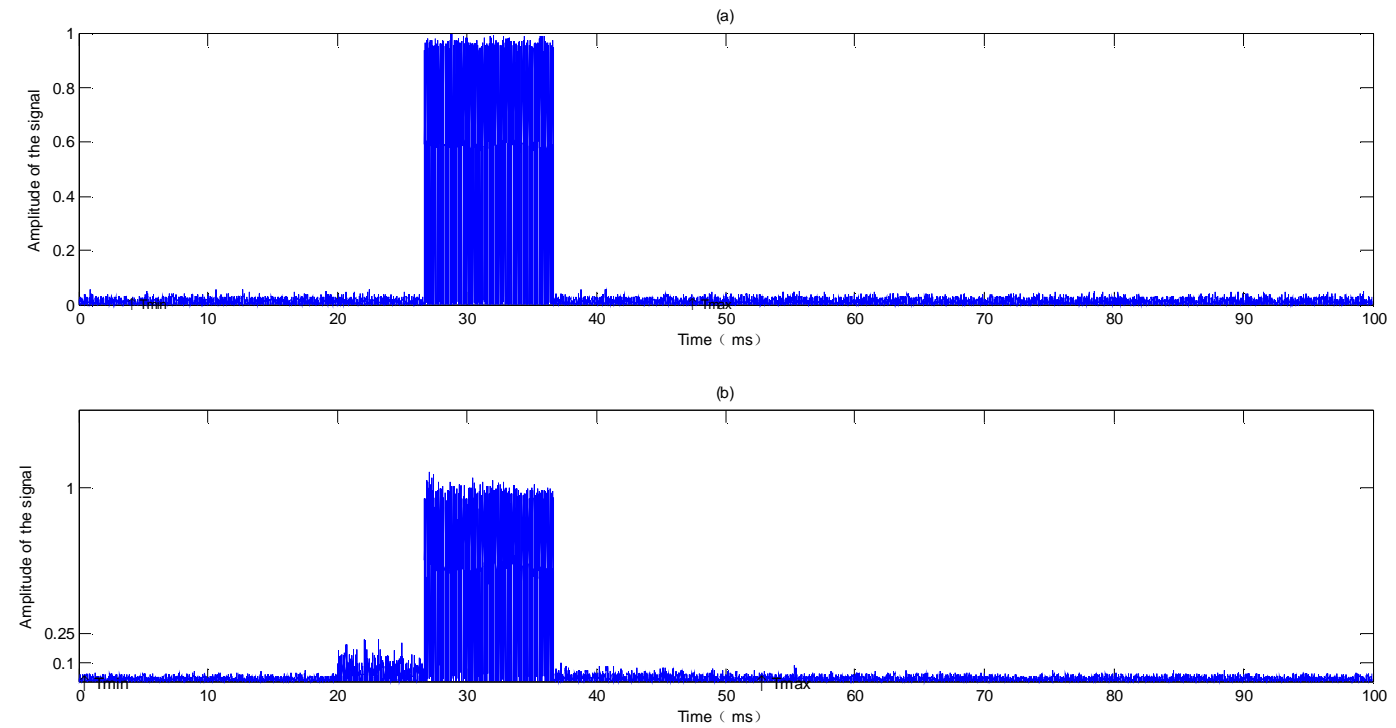

Fig. 2. Feature extraction results 


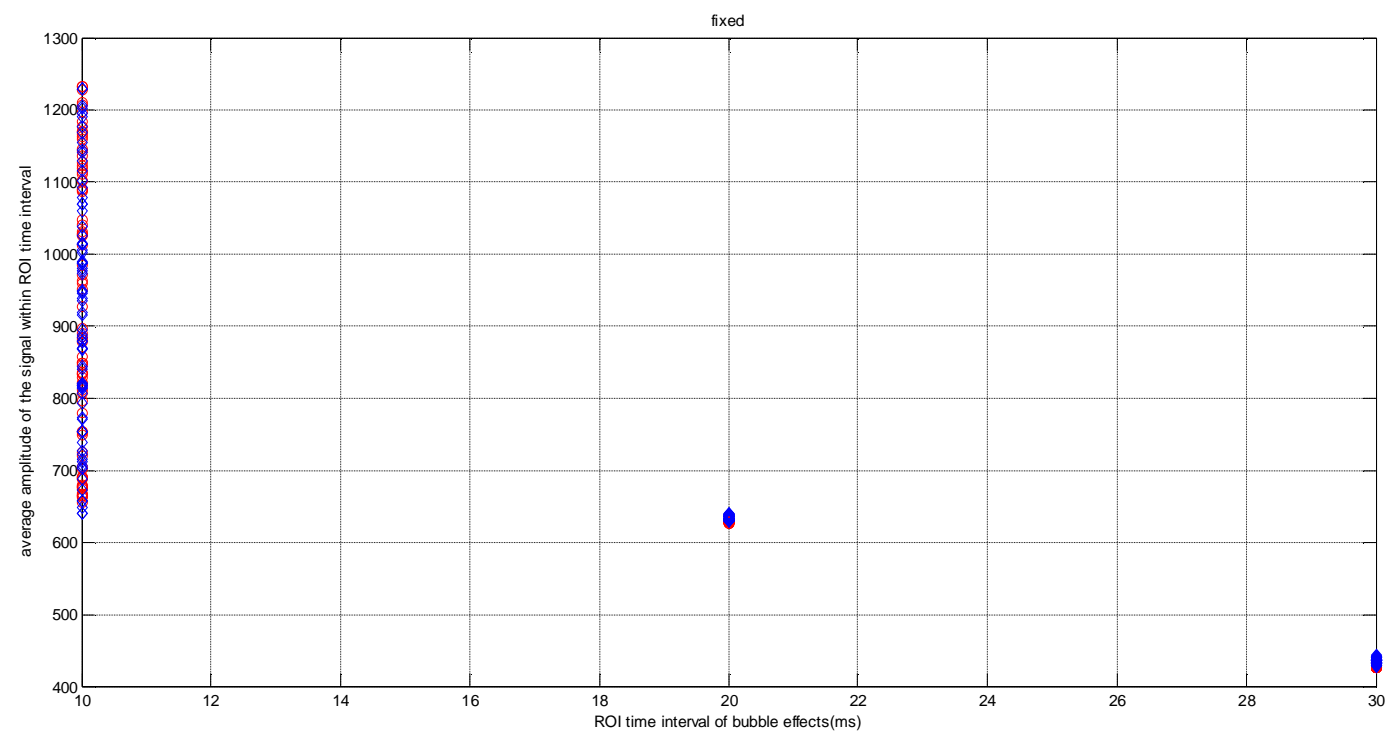

Fig. 3. The average amplitude of the pulse signals depending on the fixed ROI time interval

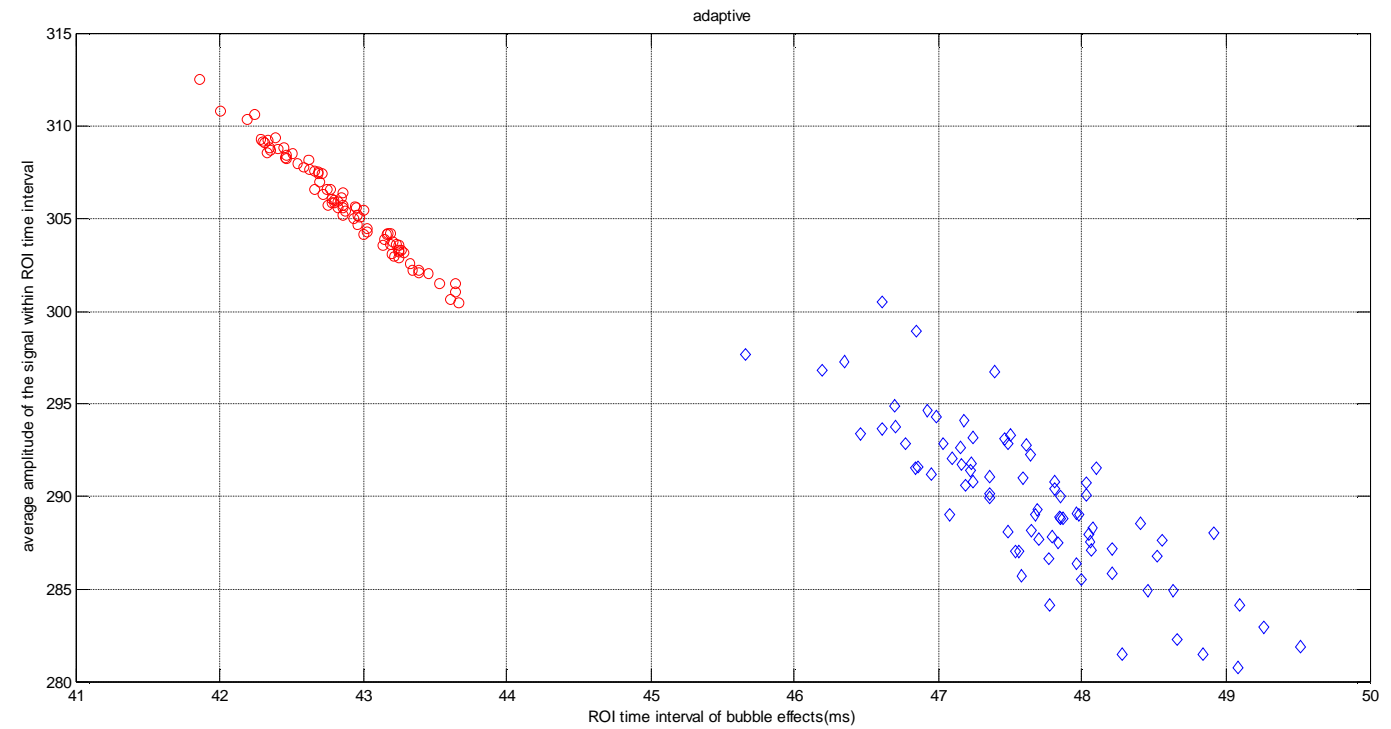

Fig. 4. Two adaptively extracted features

\section{SVM、OCSVM}

For an efficient pattern analysis of the reflected signal based on these features, a support vector machine (SVM) is used to create an optimal hyperplane, which can optimally separates the feature vectors into two nonoverlapping groups: the presence and the absence of bubble-wake signals.

The SVM model generally fails to successfully distinguish between the two classes when each number of the training data for the two classes is unbalanced. Instead, an OCSVM has a property to plausibly design an optimal detector based on properly describing a one-class data distribution in the feature space ${ }^{[4]}$. An OCSVM generates a model for representing the distribution features in the absence of bubble wake signals, which is more easily obtained than bubble-wake signals. However, due to the characteristics of signals obtained from complex real ocean environments, a nonlinear detection method needs to be considered. Applying a kernel function can transform the nonlinear problem into a linear problem by transforming the input data into a high-dimensional feature space, moreover, so we

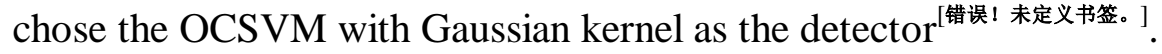


The optimization problem of an OCSVM using a kernel technique is equivalent to solving a dual quadratic programming problem as in

$$
\begin{aligned}
& \min _{\alpha} \frac{1}{2} \sum_{i, j}^{l} \alpha_{i} \alpha_{j} K\left(\mathrm{x}_{i}, \mathrm{x}_{j}\right) \\
& 0<\alpha_{i}<\frac{1}{v l}, \sum_{i}^{l} \alpha_{i}=1
\end{aligned}
$$

Based on solving this problem, the optimal nonlinear decision boundaries are obtained by

$$
\bar{f}(\mathrm{x})=\left(\sum_{i}^{l} \alpha_{i} K\left(\mathrm{x}, \mathrm{x}_{i}\right)\right)+b
$$

Gaussian kernel function is applied, as determined by

$$
K\left(x_{i}, x_{j}\right)=\exp \left(-\left\|x_{i}-x_{j}\right\|^{2} / 2 \sigma^{2}\right)
$$

Where $\sigma^{2}$ is the variance, which means the width of the Gaussian kernel function.

In order to improve the training speed, PLATT design the SMO algorithm for SVM ${ }^{[5]}$. Every step of the SMO algorithm iteration select only the Lagrange multiplier which corresponding to two data points to optimize. Its advantage is that two data points optimization analytic solution can be obtained, which means that we needn't to see quadratic optimization as part of the algorithm. Reference [6] had introduced the SMO algorithm for OCSVM

\section{Results}

This paper selects the toolbox LIBSVM to realize the ship-wake detection. LIBSVM is a support vector machine algorithm research platform developed by professor Lin Zhiren at the Taiwan University. Which provide four basic kernel function and cross validation to select parameters can effectively solve the parameters problems ${ }^{[7]}$.

When it comes to selecting of SVM parameters optimization, international has not recognized the best way to unity. We use grid search method and POS algorithm to optimize the parameters: $n$ and $\gamma=1 / \sigma^{2}$.

Linear grid search is the simplest way to find optimal model parameters. A grid structure lends itself naturally to search for discrete parameters. First of all, we select the parameter $n$ and $\gamma$ within a certain range of values. And then we set the training as a raw data set and combined with $\mathrm{K}$ fold cross-validation method, we can got the classification accuracy of training set under the fixed $n$ and $\gamma$. Take the parameter which can got the highest classification accuracy rate as the best parameters. The model parameters of contour plot is shown in Fig. 5 and the adaptive value curve is shown in Fig. 6

We generate three data sets, where data set A consisted of sixty pulse signals in the absence of a bubble wake. Data set A consist of various kinds of simulating data with 3 different SNR and 2 different thickness of the ship-wake. Meanwhile, Data set B consisted of 200 pulse signal which consisted of 100 bubble-wake pulse signals and 100 pulse signals in the absence of a bubble wake. 


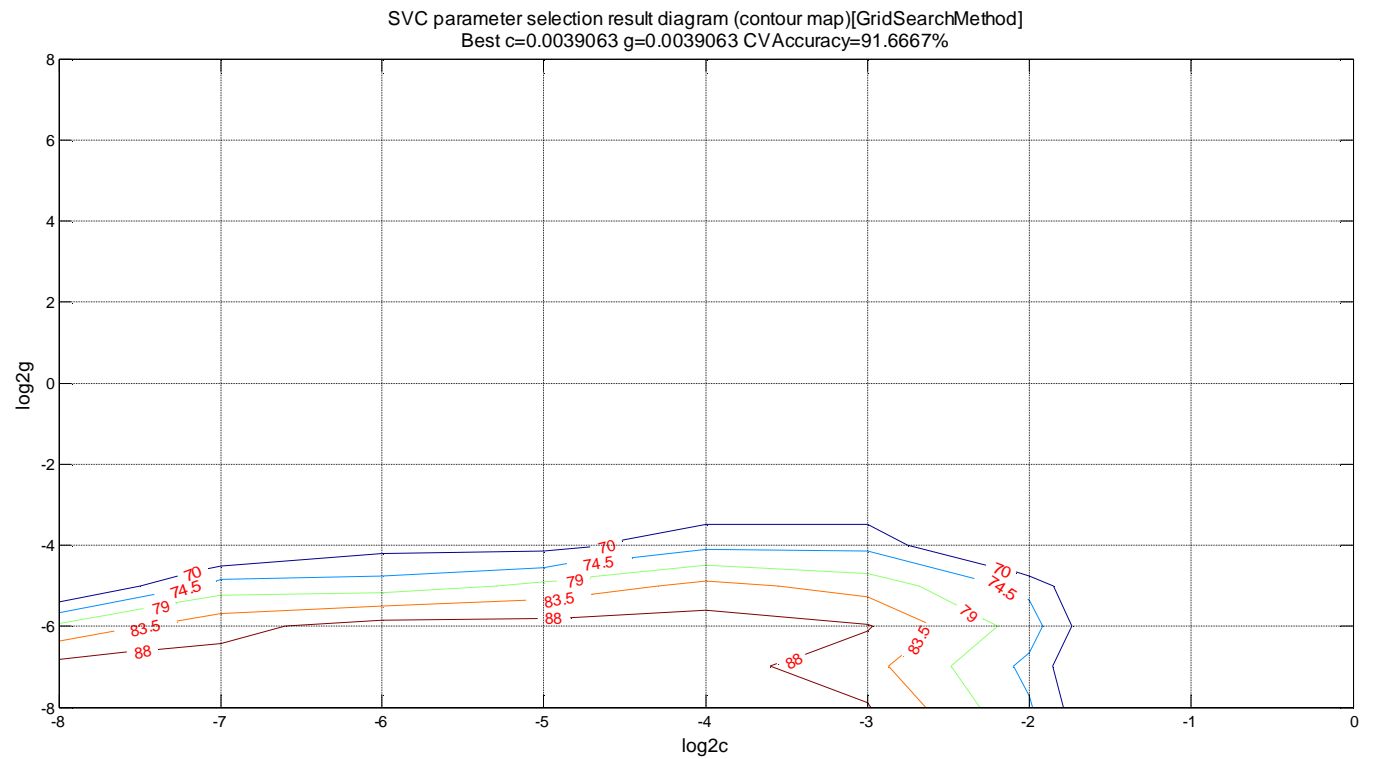

Fig. 5. Model parameters of contour plot

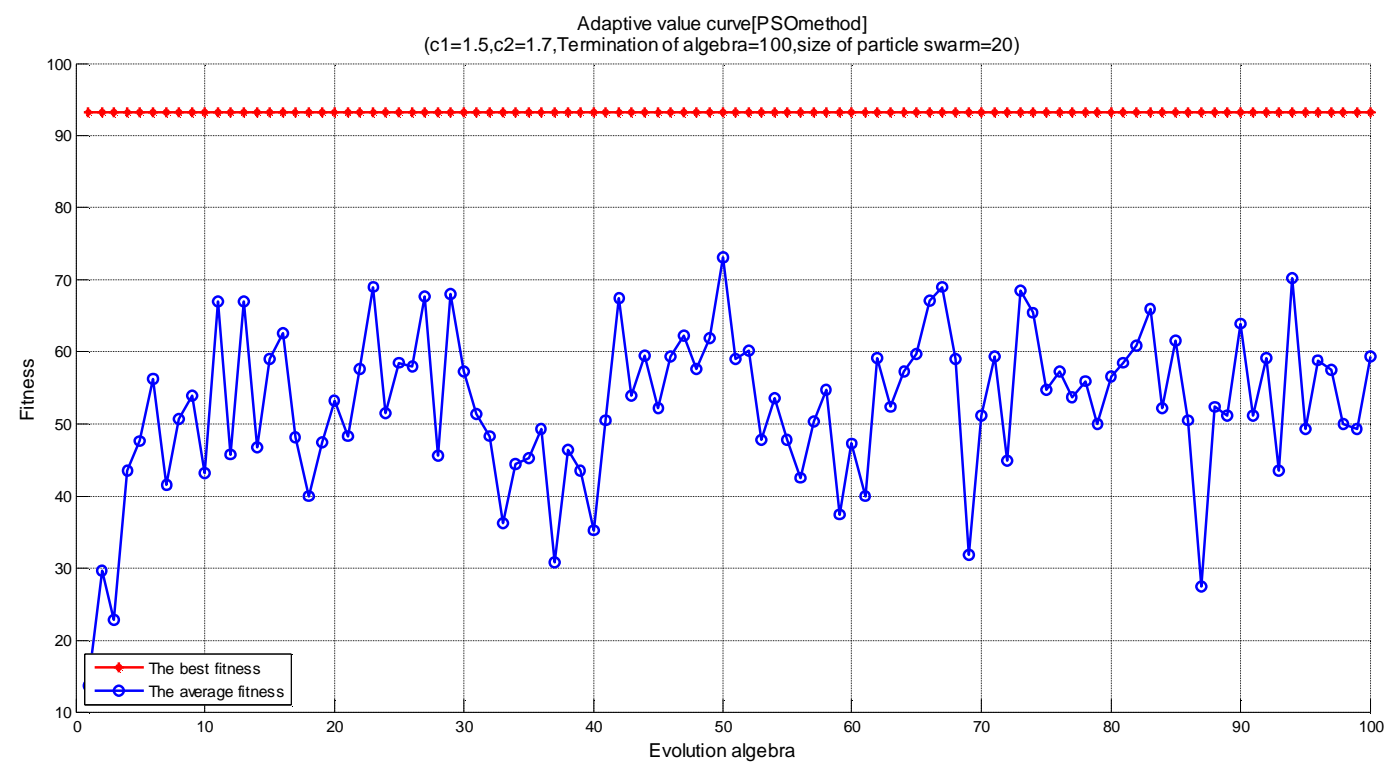

Fig. 6. Adaptive value curve

The experimental results using data set A to train the OCSVM to get the bubble-wake detector, while data set $\mathrm{B}$ are used to demonstrate the performance and robustness of the proposed OCSVM-based detector. We divide data set $\mathrm{C}$ evenly into 5 parts, and classify these pulse signals respectively, the average the five detection results is shown in Table I. Change the SNR and the thickness of the ship wake and repeat the above operation.

Table I and Table II shows the mean of the detection results when using data set B with different SNR. The proposed model was able to detect the bubble-wake signals perfectly with 40 and 20dB SNR signals. However, with $10 \mathrm{~dB}$ SNR signals, the detecting precision is reduced sharply but still reasonable, since these signals were too noisy to be discriminated against, even by a human.

Compared with Table I and Table II, the detecting precision of the proposed model improved as the thickness of the bubble wake increased. The result is consistent with the actual situation that the thicker the ship-wake is, the easy to be detected. Both of grid search method and POS algorithm can get the can find good parameters, and POS algorithm is a little better than grid search. 
TABLE I. THICKNESS OF WAKE=5M

\begin{tabular}{c|cccc}
\hline SNR/dB & $\mathbf{4 0}$ & $\mathbf{3 0}$ & $\mathbf{2 0}$ & $\mathbf{1 0}$ \\
\hline Grid search /\% & 93.75 & 97.00 & 97.25 & 73.75 \\
\hline POS method /\% & 93.50 & 100 & 98.50 & 76.25 \\
\hline
\end{tabular}

TABLE II. THICKNESS OF WAKE=8M

\begin{tabular}{c|cccc}
\hline SNR/dB & $\mathbf{4 0}$ & $\mathbf{3 0}$ & $\mathbf{2 0}$ & $\mathbf{1 0}$ \\
\hline Grid search /\% & 93.75 & 96.00 & 99.50 & 74.50 \\
\hline POS method /\% & 93.75 & 100 & 99.75 & 79.75 \\
\hline
\end{tabular}

\section{Conclusion}

Based on the acoustics characteristic of ship wake signal, the modeling and simulating methods of the mathematic wake echo signal are designed. We extract features adaptively from the simulated echo signal and then use the modified OCSVM based on SMO method with a kernel function to detect the ship wake. And the grid search method and POS algorithm were used to search optimal parameter. The efficiency and the robustness of the proposed method for the whole system design were verified.

\section{References}

[1] LI Ting , LIU Chun - w u, HUANG Zhi - ping, GUO Xi - y e,"Modeling and Simulating of Ship Wake Echo Signal,’Computer Simulation, vol. 28, NO. 6, pp. 29, Jun, 2011.

[2] L. M. Manevitz and M. Yousef, "One-class SVMs for document classification," J. Mach. Learn. Res., vol. 2, pp. 139-154, 2001.

[3] Sungmoon Jeong, Sang-Woo Ban, Sangmoon Choi, Donghun Lee, and Minho Lee, "Surface Ship-Wake Detection Using Active Sonar and One-Class Support Vector Machine," IEEE Journal Of Oceanic Engineering, VOL. 37, NO. 3, pp. 456-466, JULY 2012

[4] Zhao Wei, "Acoustic Model Establishment of Ship Wake Echo Waveform for Self-guided Torpedo," Ship Electronic Engineering, vol. 33. No. 4, pp. 81-83, 2013

[5] PLATT J. Sequential minimal optimization: A fast algorithm for training support vector machines [R]. Technical Report MSR-TR-98-14, Microsoft Research, 1998

[6] Hu Lei, HU Niao-qing, QIN Guo-jun, "Support vector machines detection method for turbopump test data analysis," Journal Of Propulsion Technology, vol. 129, No. 12, pp. 244-248, Apri1 2008

[7] Wu Hao, LI Qun-zhan, LIU Wei, "A new algorithm of wide-area backup protection based on PSO-LIBSVM" Power System Protection and Control, vol. 41, No. 15, pp. 49-58, Aug. 1. 2013

[8] Jun Tang,, Xiaojuan Zhao, "A Hybrid Particle Swarm Optimization with Adaptive Local Search," Journal Of Networks, vol. 5, No. 4, pp .411-418, APRIL 2010 